\title{
Maximum Battery Life Routing to Support Ubiquitous Mobile Computing in Wireless Ad Hoc Networks
}

\author{
C.-K. Toh, Georgia Institute of Technology
}

\begin{abstract}
Most ad hoc mobile devices today operate on batteries. Hence, power consumption becomes an important issue. To maximize the lifetime of ad hoc mobile networks, the power consumption rate of each node must be evenly distributed, and the overall transmission power for each connection request must be minimized. These two objectives cannot be satisfied simultaneously by employing routing algorithms proposed in previous work. In this article we present a new power-aware routing protocol to satisfy these two constraints simultaneously; we also compare the performance of different types of power-related routing algorithms via simulation. Simulation results confirm the need to strike a balance in attaining service availability performance of the whole network vs. the lifetime of ad hoc mobile devices.
\end{abstract}

\section{INTRODUCTION}

Military, law enforcement, and disaster relief operations are often carried out in situations with no preexisting network infrastructure (e.g., base stations). Although one could install temporary base stations to provide wireless coverage, this results in a single point of failure in a hostile environment, and is therefore undesirable from a reliability standpoint. A robust solution is to allow various hosts to organize themselves into a network, known as an ad hoc mobile wireless network. Ad hoc networks can operate without the presence of static radio base stations, unlike cellular wireless networks.

The first part of this article presents the salient characteristics of an ad hoc wireless network. Since existing wired routing protocols cannot be used directly in an ad hoc wireless network environment, different routing schemes should be proposed for ad hoc networks. The performance of an ad hoc wireless network is dependent on the routing algorithm or protocol employed. Therefore, our second objective is to highlight the desirable properties of ad hoc routing protocols.
Unlike the cellular wireless network, several mobile hosts need to be present in order to organize themselves into an ad hoc wireless network.

The lack of mobile hosts can result in partitioning of the network, causing interruptions in communications between mobile hosts. Since most mobile hosts today are powered by batteries, efficient utilization of battery power is more important than in cellular networks. It also has an important influence on the overall communication performance of the network. Our third objective is to examine, propose, and compare different power-efficient ad hoc routing schemes. Lastly, we present a conclusion on our findings.

\section{Characteristics OF Ad HoC MOBILE WIRELESS NETWORKS}

An ad hoc mobile network is an autonomous system consisting of mobile hosts that do not rely on the presence of any fixed network infrastructure. Depending on the nodes' geographical positions, their transceiver coverage patterns, transmission power levels, and co-channel interference levels, a network can be formed and unformed on the fly. This ad hoc network topology changes as mobile hosts migrate, "disappear" (failure or depletion of battery capacity), or adjust their transmission and reception characteristics. The main characteristics of ad hoc networks are:

- Dynamic topology: Nodes are free to move about arbitrarily. In addition, radio propagation conditions change rapidly over time. Thus, the network topology may change randomly and rapidly over unpredictable times.

- Bandwidth constraints and variable link capacity: Wireless links have significantly lower capacity than wired links. Due to the effects of multiple access, multipath fading, noise, and signal interference, the capacity of a wireless link can be degraded over time and the effective throughput may be less than the radio's maximum transmission capacity.

- Energy constrained nodes: Mobile nodes 
rely on batteries for proper operation. Since an ad hoc network consists of several nodes, depletion of batteries in these nodes will have a great influence on overall network performance. Therefore, one of the most important protocol design factors is related to device energy conservation.

- Multi-hop communications: Due to signal propagation characteristics of wireless transceivers, ad hoc networks require the support of multihop communications; that is, mobile nodes that cannot reach the destination node directly will need to relay their messages through other nodes.

- Limited security: Mobile wireless networks are generally more vulnerable to security threats than wired networks. The increased possibility of eavesdropping, spoofing, and denial-of-service (DoS) attacks should be carefully considered when an ad hoc wireless network system is designed.

To support mobile computing in ad hoc wireless networks, a mobile host must be able to communicate with other mobile hosts, which may not lie within its radio transmission range. To support ad hoc mobile communications under the influence of the above-mentioned factors, an ad hoc routing protocol will need to perform four functions, namely:

- Determining/detecting changing network topology

- Maintaining network topology/connectivity

- Scheduling of packet transmission and channel assignment

- Routing

We shall now examine each of these functions.

\section{Determination of Network Topology}

An ad hoc routing protocol must determine and monitor the dynamics of network topology over time. Since multihop communications are needed in ad hoc networks, the routing protocol must ensure that links in the route have strong connectivity. There must exist at least one path from any node to any other node in a nonpartitioned network. A node in the ad hoc network must be aware of its surrounding environment and the neighboring nodes with which it can directly communicate. It must take into account the relative difficulties of forming links with those nodes and the benefits such connections will bring to the network on the whole (e.g., bandwidth usage, transmission delay, throughput, power consumption). Basically, there are two approaches in providing ad hoc network connectivity:

- Flat-routed network architecture

- Hierarchical network architectures [1]

In the flat-routed network architecture, all the nodes are equal and packet routing is done based on peer-to-peer connections. However, in hierarchical networks, at least one node in each lower layer is designated to serve as a gateway or coordinator to higher layers.

\section{Maintaining NetWork Connectivity Under Changing Radio Conditions and Mobility}

Since the location of each mobile host can change over time, the network topology also changes frequently as a result. Topological changes can occur due to:

- The breakdown of a mobile host in a hostile environment

- The failure of a connected link due to signal interference and changes in signal propagation conditions

Therefore, an ad hoc routing protocol must be able to dynamically update the status of its links and reconfigure itself in order to maintain strong connectivity to support communications among the nodes. Any centralized algorithm proposed to maintain the network topology is vulnerable (since the central node could also move) and it takes too much time and effort to disseminate node/link change information to all nodes. Therefore, a fully distributed algorithm that provides reliability and robust operation against topological changes and component (mobile hosts, links) failures is preferred.

\section{TRANSMISSION SCHEDULING AND Channel Assignment}

Since a new radio transmission by a mobile host may affect an existing communication link through signal interference, an efficient packet scheduling and channel assignment algorithm is needed to ensure that the new transmissions will not conflict with an existing one. In addition, it will ensure efficient use of the limited available bandwidth. In a single-channel radio system, transmissions over the wireless media should be adequately scheduled in order to maintain sufficient spatial distances among the nodes. This can lead to the reduction of interference among the links. In a multichannel radio system, the channel assignment algorithm can help to reduce interference among transmissions occurring over the same channel, thus decreasing the possibility of affecting an existing transmission and wasting bandwidth.

\section{PACKet ROUTING}

Compared to wired networks with static nodes, ad hoc networks require a highly adaptive routing scheme to cope with the high rate of topology changes. This implies that the routing protocol should propagate topology changes and compute updated routes to the destination. In general, existing routing protocols can be classified as either:

- Table-driven

- On-demand

- Hybrid $[1,2]$

Table-driven protocols attempt to continuously update the routes within the network so that when a packet needs to be forwarded, the route is already known and can immediately be used. The family of distance-vector or link-state algorithms are examples of table-driven schemes. On the other hand, on-demand schemes invoke a route discovery procedure only on a need basis. Thus, when a route is needed, some sort of global or localized search procedure is employed.

\section{Desired Properties of Ad Hoc Routing PROTOCOLS}

In the previous section we summarize the required protocol functions for ad hoc wireless

In a multichannel
radio system,
the channel
assignment
algorithm can
help to reduce
interference
among
transmissions
occurring over
the same
channel, thus
decreasing the
possibility of
affecting an
existing
transmission and
wasting
bandwidth.

In a multichannel

radio system

can

interference

among

transmissions

the same

channel, thus

decreasing the

possibility of

affecting an

width. 


To prolong the
lifetime of each
node, ad hoc
routing protocols
should consider
power
consumption.
For example,
routing protocols
should be able to
accommodate
sleep periods
without causing
any adverse
consequences.

networks. Although there are many, we shall focus on routing protocols for ad hoc wireless networks. The desirable properties of ad hoc routing protocols are discussed below.

\section{DistRIBUTED IMPLEMENTATION}

Since ad hoc wireless networks are autonomous and self-organizing systems, routing protocols must be distributed in nature without relying on centralized authorities.

\section{EfFicient Utilization OF BANDWIDTH}

If a routing protocol incurs excessive control traffic, the available network bandwidth will be consumed by control traffic. This can impact communication performance. Since the bandwidth of a wireless network is limited, reduction of control overhead is an important design factor. For example, table-driven ad hoc routing protocols propagate routing information (distance-vector or link-state tables) periodically, incurring significant control overhead.

\section{EfFiCiEnt Utilization OF BATTERY CAPACITY}

Unlike cellular networks, the lifetime of mobile hosts will deeply impact the performance of the ad hoc mobile network. In a cellular network, a reduction in the number of active mobile users will reduce the amount of signal interference and channel contentions. However, since ad hoc mobile hosts need to relay their messages through other hosts toward their intended destinations, a decrease in the number of mobile users can also degrade network performance. As the number of available hosts decreases, the network may also be partitioned into smaller networks. To prolong the lifetime of each node, ad hoc routing protocols should consider power consumption. For example, routing protocols should be able to accommodate sleep periods without causing any adverse consequences; that is, hosts can stop transmitting and/or receiving for arbitrary periods of time when it is ideal. Moreover, transmission power can be used as a routing metric.

\section{Optimization of Metrics}

End-to-end throughput and delay are widely used performance metrics in wired and wireless networks. However, since the network topology is dynamically changing, the bandwidth and battery power are important factors in wireless ad hoc networks. Hence, we should also consider other metrics as well. Such metrics can influence the design of routing protocols, and there exist trade-offs in using different metrics. For example, although on-demand routing algorithms can reduce control overhead (i.e., optimizing the bandwidth), it requires some route acquisition time (i.e., the time required to discover and establish a route when desired), thus increasing end-to-end delay. Therefore, in routing protocol design one should optimize some reasonable metrics in addition to others. The following is a list of metrics worthy of consideration:

- Maximum end-to-end throughput

- Minimum end-to-end delay

- Shortest path/minimum hop

- Minimum total power (battery capacity)

- Load balancing (least congested path)

- Minimum overhead (bandwidth)
- Adaptability to the changing topology

- Association stability [3] (i.e., longevity of routes)

- Route relaying load [3]

\section{FASt ROUTE CONVERGENCE}

Since the network topology dynamically changes, routing protocols should provide a new and stable route as soon as possible after a topology change. For example, in distance-vector routing, the speed of convergence to a new route is slow and may provide inaccurate route information to the destination due to presence of stale routes. Fast route convergence is achieved by requiring nodes to frequently broadcast route updates, which is not efficient in a wireless ad hoc environment.

\section{FREEDOM FROM LOOPS}

The paths derived from the routing tables of all nodes should not have loops. Some routing algorithms can cause temporary loops (i.e., a small fraction of packets spinning around the network for an arbitrary period of time). Looping of packets can result in considerable overhead in terms of bandwidth and power consumption. Using time-to-live (TTL) values can help mitigate this problem, but a more structured and well-thought-out approach is desirable because it can lead to better communication performance.

\section{UNIDIRECTIONAL LINK SUPPORT}

Bidirectional links are typically assumed in the design of routing algorithms. However, there are a number of factors that will cause wireless links to be unidirectional, including the presence of different radio capabilities and signal interference. Therefore, in situations where a pair of unidirectional links (in opposite directions) form the only bidirectional connection, the ability to make use of unidirectional links is considered valuable.

\section{POWER-EFfiCIENT Ad HOC MOBILE Networks}

\section{Efficient Utilization of Battery Power}

Since most mobile hosts of an ad hoc network today operate using batteries, it is important to minimize the power consumption of the entire network (implying maximizing the lifetime of ad hoc networks). The power required by each mobile host can be classified into two categories:

- Communication-related power

- Non-communication-related power

The former can be further divided into two parts, namely:

- Processing power

- Transceiver power

Each mobile host spends some processing power to execute network algorithms and run applications. Transceiver power refers to the power used by the radio transceiver to communicate with the other mobile hosts. In mobile power consumption, each protocol layer is closely coupled. For example, if a routing protocol requires frequent updates of routing information, it is difficult to implement sleep mode at the data link layer. We therefore briefly summarize the power conservation schemes for each layer below. 
Physical Layer and Wireless Device - Currently, research has been done for hardware and circuit-level power optimization and management within a wireless device (e.g., low-power displays, low-power CPUs, power-efficient computing algorithms). Within a single wireless device, improved power efficiency may be achieved for conventional components (e.g., CPU and disk) by turning them off or slowing them down when not needed [4].

At the physical layer, transmission power can be adjusted. The use of excessive transmission power can increase the interference to other hosts and will cause an increase in transmission power by other hosts. Therefore, physical layer functions should include transmitting data at the minimum power level to maintain links, and adapt to changes in transmission environment (due to host mobility or co-channel interference) [5]. Summarizing, power control can maintain a link at the minimum power level, but can also prolong an existing link against interference by increasing the transmission power.

Data Link Layer - At the data link layer, energy conservation can be achieved by using effective retransmission request schemes and sleep mode operation. The data link layer provides error-free communication between two nodes. It detects transmission errors and then retransmits the correct packet using automatic repeat request (ARQ). In ad hoc networks, due to the presence of mobility and co-channel interference, transmission errors can occur frequently, which lead to frequent retransmission requests. Since retransmissions increase power consumption and cause higher interference to other users, a new efficient retransmission request scheme is needed for ad hoc networks.

One possible scheme is the following: when a transmitter does not receive acknowledgments after packet transmissions, the retransmission request scheme senses that the channel is bad and ceases retransmitting to not waste power when the chances of successful reception are $\operatorname{dim}$ [4]. This scheme, therefore, reduces unnecessarily power wastage at the expense of transmission delay. Another solution is for the retransmission request scheme to increase the retransmission power. This reduces the possibility of transmission errors but increases the signalto-interference ratio (SIR) of the network. Therefore, to appropriately determine when and at what power level a mobile host should attempt retransmission is an important issue for minimizing power incurred at the data link layer [5].

A node transmitting packets to its downstream nodes will be overheard by all neighboring nodes. Hence, all neighboring nodes will consume power even though the packet transmission was not directed to them [6]. Therefore, to reduce power consumption a node's transceiver should be powered off (snooze or not listening) when not in use.

Network Layer - In wired networks, the emphasis has traditionally been on maximizing end-to-end throughput and minimizing delay. In general, paths are computed based on minimizing hop count or delay. Nonetheless, to maxi- mize the lifetime of mobile hosts, routing algorithms must select the best path from the viewpoint of power constraints as part of route stability. Hence, routes requiring lower levels of power transmission are preferred, but this can affect end-to-end throughput.

Transmission with higher power increases the probability of successful transmission, thus increasing end-to-end throughput. However, it also yields higher interference to other mobile hosts, which can destroy an existing transmission band and may cause the network to have blocked calls. This could result in a decrease in network capacity [7]. Therefore, lower power transmission does not always have a negative impact on throughput. Since lower power transmission can reduce channel interference and contentions, it can increase end-to-end throughput. When power efficiency is considered, ad hoc networks will require a routing algorithm that can evenly distribute packet-relaying loads to each node to prevent nodes from being overused or abused. By maximizing the lifetime of all nodes, the time before the network is partitioned is prolonged.

\section{Power-Efficient Ad Hoc Routing}

\section{Power-Efficient Routing Protocols}

In the previous section we summarize the schemes to reduce power consumption at each protocol layer. At the network layer, routing algorithms must select the best path to minimize the total power needed to route packets on the network and maximize the lifetime of all nodes. We shall present four variations of route selection schemes to achieve one or both of these goals.

\section{Minimum Total Transmission Power Routing} (MTPR) - In wireless communications, radio propagation can be modeled effectively with a $1 / d^{n}$ transmit power rolloff (usually, $n=2$ for short distance and $n=4$ for longer distance). For successful transmissions, the signal-to-noise ratio (SNR) received at a host $n_{j}$ should be greater than a specified predetection threshold $\psi_{j}$. This threshold $\psi_{j}$ is closely related to the bit error rate (BER) of the received signal. For successful transmissions from a host $n_{i}$ to $n_{j}$, the SNR at host $n_{j}$ should satisfy the following equation:

$$
S N R_{j}=\frac{P_{i} G_{i, j}}{\sum_{k \neq i} P_{K} G_{k, j}+\eta_{j}} \Psi_{j}(B E R),
$$

where $P_{i}$ is the transmission power of host $n_{i}, G_{i, j}$ is the path gain between hosts $n_{i}$ and $n_{j}$ (i.e., $G_{i, j}$ $\left.=1 / d_{i j}^{n}\right)$, and $\eta_{j}$ is the thermal noise at host $n_{j}$.

Therefore, the minimum transmission power is dependent on interference noise, distance between hosts, and desired BER. To obtain the route with the minimum total power, the transmission power $P\left(n_{i}, n_{j}\right)$ between hosts $n_{j}$ and $n_{j}$ can be used as a metric [6]. The total transmission power for route $l, P_{l}$, can be derived from

$$
P_{l}=\sum_{i=0}^{D-1} P\left(n_{i}, n_{n+1}\right) \quad \text { for all node } n_{i} \in \text { route, }
$$

where $n_{0}$ and $n_{D}$ are the source and destination nodes, respectively. Therefore, the desired route
To maximize the

lifetime of mobile

hosts, routing

algorithms must

select the best

path from the viewpoint of

power constraints

as part of route

stability. Hence,

routes requiring

lower levels of

power

transmission are

preferred, but

this can affect end-to-end

throughput. 
The basic idea

behind CMMBCR

is that when all

nodes in some

possible routes

between a source

and a destination

have sufficient

remaining battery

capacity, then a

route with

minimum total

transmission

power among

these routes is

chosen. $k$ can be obtained from

$$
P_{k}=\min _{l \in A} P_{l},
$$

where $A$ is the set containing all possible routes.

The above function can be solved by a standard shortest path algorithm such as Dijkstra or Bellman-Ford. In [7], Dijkstra's shortest path algorithm was modified to obtain the minimum total power route. However, since transmission power depends on distance ${ }^{1}$ (proportional to $d^{n}$ ), this algorithm will select routes with more hops than other routing algorithms. In general, the more nodes involved in routing packets, the greater the end-to-end delay. In addition, a route consisting of more nodes is more likely to be unstable, because the probability that intermediate nodes will move away is higher. Hence, from the standpoint of minimum hops, the route obtained from the above algorithm is not attractive.

To overcome this problem, transceiver power (the power used when receiving data) as well as transmission power were considered as a cost metric, and the distributed Bellman-Ford algorithm was used [8]. At node $n_{j}$, it computes

$$
\begin{aligned}
C_{i, j}= & P_{\text {transmit }}\left(n_{i}, n_{j}\right)+P_{\text {transceiver }}\left(n_{j}\right) \\
& +\operatorname{Cost}\left(n_{j}\right),
\end{aligned}
$$

where $n_{i}$ is a neighboring node of $n_{j}$, $P_{\text {transceiver }}\left(n_{j}\right)$ is the transceiver power at node $n_{j}$, and $\operatorname{Cost}\left(n_{j}\right)$ is the total power cost from the source node to node $n_{j}$. This value is sent to node $n_{i}$. Subsequently, at node $n_{i}$ it computes its power cost by using the following equation:

$$
\begin{aligned}
& \operatorname{Cost}\left(n_{i}\right)=\min _{j \in N H(i)} C_{i, j}, \text { where } \\
& N H(i)=\left\{j ; n_{j} \text { is a neighbor node of } n_{i}\right\} .
\end{aligned}
$$

The path with minimum cost from the source node to node $n_{i}$ is selected. This procedure is repeated until the destination node is reached. In this algorithm, $P_{\text {transceiver }}\left(n_{j}\right)$ helps the algorithm find routes with fewer hops than the MTPR algorithm because generally the transceiver power is identical for hosts using the same transceiver.

Minimum Battery Cost Routing (MBCR) Total transmission power is an important metric because it concerns the lifetime of mobile hosts. However, it has a critical disadvantage. Although this metric can reduce the total power consumption of the overall network, it does not reflect directly on the lifetime of each host. If the minimum total transmission power routes are via a specific host, the battery of this host will be exhausted quickly, and this host will die of battery exhaustion soon. Therefore, the remaining battery capacity of each host is a more acurate metric to describe the lifetime of each host [8].

Let $c_{i}^{t}$ be the battery capacity of a host $n_{i}$ at time $t$ ranging between 0 and 100 . We define $f_{i}\left(c_{i}^{t}\right)$ as a battery cost function of a host $n_{i}$. Now, suppose a node's willingness to forward packets is a function of its remaining battery capacity. The less capacity it has, the more reluctant it is.

${ }^{1}$ Assuming that each node may have different transmission power.
As proposed, one possible choice for $f_{i}$ is

$$
f_{i}\left(c_{i}^{t}\right)=\frac{1}{c_{i}^{t}} .
$$

As the battery capacity decreases, the value of cost function for node $n_{i}$ will increase. The battery cost $R_{j}$ for route $i$, consisting of $D$ nodes, is

$$
R_{j}=\sum_{i=0}^{D_{j}-1} f_{i}\left(c_{i}^{t}\right)
$$

Therefore, to find a route with the maximum remaining battery capacity, we should select a route $i$ that has the minimum battery cost.

$$
R_{i}=\min \left\{R_{j} \mid j \in A\right\},
$$

where $A$ is the set containing all possible routes.

Since battery capacity is directly incorporated into the routing protocol, this metric prevents hosts from being overused, thereby increasing their lifetime and the time until the network is partitioned [8]. If all nodes have similar battery capacity, this metric will select a shorter-hop route. However, because only the summation of values of battery cost functions is considered, a route containing nodes with little remaining battery capacity may still be selected. For example, in Fig. 1 there are two possible routes between the source and destination nodes. Although node 3 has much less battery capacity than other nodes, the overall battery cost for route 1 is less than route 2 . Therefore, route 1 will be selected, reducing the lifetime of node 3 , which is undesirable.

Min-Max Battery Cost Routing (MMBCR) To make sure that no node will be overused, the above objective function (Eq. 3) can be modified, as indicated in [8]. Battery cost $R_{j}$ for route $j$ is redefined as

$$
R j=\max _{i \in \text { route }_{-} j} f_{i}\left(c_{i}^{t}\right) .
$$

Similarly, the desired route $i$ can be obtained from the equation

$$
R_{i}=\min \left\{R_{j} \mid j \in A\right\} .
$$

Since this metric always tries to avoid the route with nodes having the least battery capacity among all nodes in all possible routes, the battery of each host will be used more fairly than in previous schemes. Initially, it seems that the lifetime of all nodes will be elongated. However, on closer examination, since there is no guarantee that minimum total transmission power paths will be selected under all circumstances, it can consume more power to transmit user traffic from a source to a destination, which actually reduces the lifetime of all nodes.

Our Proposed Conditional Max-Min Battery Capacity Routing - According to previous discussions, our goal is to maximize the lifetime of each node and use the battery fairly. However, these two goals cannot be achieved simultaneously by applying MTPR or MMBCR schemes. MBCR can only fulfill both of them sometimes. $^{2}$ It is still not clear at this stage if we can achieve these two goals simultaneously. To resolve this problem, we use battery capacity 
instead of cost function as a route selection metric, and introduce the conditional max-min battery capacity routing (CMMBCR) scheme.

The basic idea behind CMMBCR is that when all nodes in some possible routes between a source and a destination have sufficient remaining battery capacity (i.e., above a threshold), a route with minimum total transmission power among these routes is chosen. Since less total power is required to forward packets for each connection, the relaying load [3] for most nodes will be reduced, ${ }^{3}$ and their lifetime will be extended. However, if all routes have nodes with low battery capacity (i.e., below a threshold), routes including nodes with the lowest battery capacity should be avoided to extend the lifetime of these nodes. We define the battery capacity $R_{j}^{c}$ for route $j$ at time $t$ as

$$
R_{j}^{c}=\min _{i \in \text { route_j }} c_{i}^{t} .
$$

Let $A$ be a set containing all possible routes between any two nodes at time $t$ and satisfying the following equation:

$$
R_{j}^{c} \geq \gamma, \text { for any route } j \in A \text {. }
$$

$\gamma$ is a threshold and ranges between 0 and 100 . Let $\mathrm{Q}$ denote the set containing all possible paths between the specified source and destination nodes at time $t$. Then we arrive at:

- If $A \cap \mathrm{Q} \neq \Phi$, which implies that all nodes in some paths have remaining battery capacity higher than $\gamma$, choose a path in A $\cap \mathrm{Q}$ by applying the MTPR scheme.

- Otherwise, select route $i$ with the maximum battery capacity: $R_{i}^{c}=\max \left\{R_{j}^{c} \mid j \in Q\right\}$.

If $\gamma=0$, Eq. 6 is always true, and this metric is identical to MTPR. If $\gamma=100$, Eq. 6 is always false, and this metric is identical to MMBCR because at this time, routes with less battery capacity will always be avoided. $\gamma$ can be viewed as a protection margin. If some nodes' battery capacity goes below this value, they will be avoided to elongate lifetime. The performance of CMMBCR will therefore depend on the value of $\gamma$.

\section{Performance of DifFERENT ROUTING AlgORITHMS CONSIDERING POWER EFFICIENCY}

\section{THE STRUCtURE OF OUR Simulator}

Different routing protocols have been proposed for ad hoc wireless networks. Some use conventional routing metrics such as minimum hop, delay, and fast adaptability, while others consider new routing metrics such as stability, load balancing, and power consumption. To better understand their performance in terms of power

2 If each node uses the same transmission power, and all nodes have similar battery capacity, then $M B C$ can find a shorter path (i.e. lower total transmission power).

${ }^{3}$ To further reduce the relaying load, nodes' association stability must be taken into consideration. This require further study.

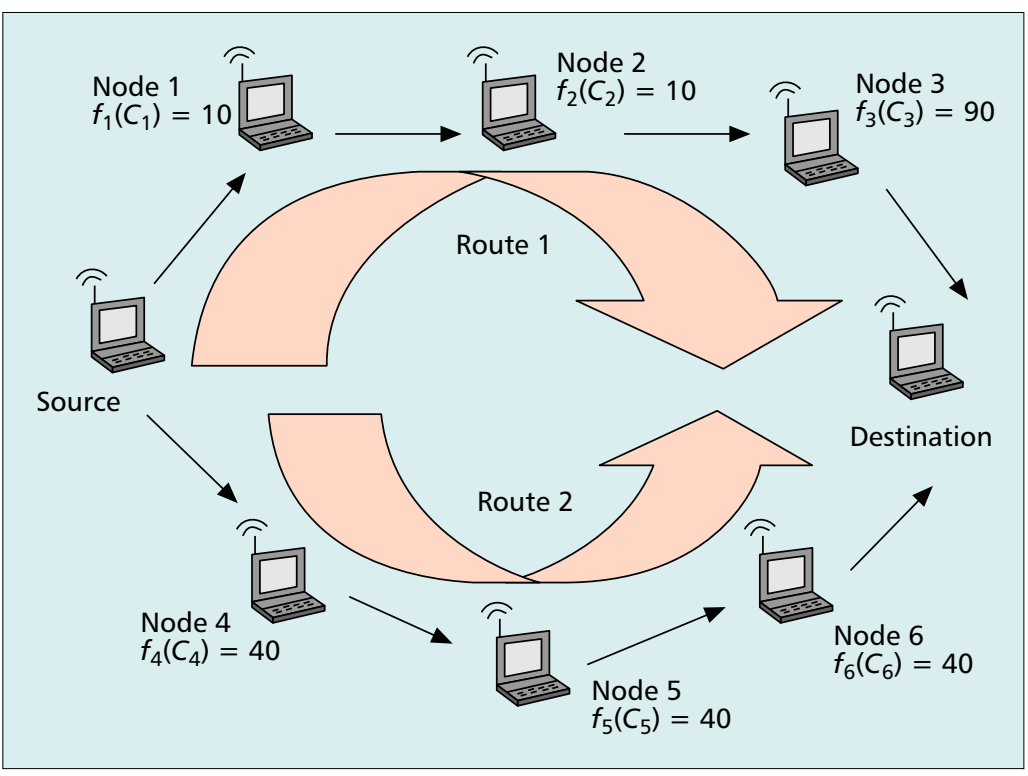

Figure 1. An illustration of the shortcoming in minimum-hop routing.

efficiency, we perform simulations. Our simulator has five major components:

- Ad hoc mobile network generator

- Mobile host migration engine

- Call/route event generator

- Routing protocol

- Power consumption computation module (Fig. 2)

Ad hoc Mobile Network Formation - Thirty mobile hosts are randomly distributed in a confined space of $100 \mathrm{~m} \times 100 \mathrm{~m}$. Each mobile host has a wireless cell size of $25 \mathrm{~m}$ radius.

Mobile Host Migration Engine - At the beginning of each simulation time slot, each mobile host randomly chooses a new direction and moves a distance equal to the product of its speed and the length of a time slot. If it reaches the boundary of the confined space, it bounces back. Each node moves at a speed of $2 \mathrm{~m} / \mathrm{s}$.

Route Requests Event Generator - Route requests are generated according to a Poisson process. When a request occurs, two nodes are randomly selected as source and destination. The request arrival rate is proportional to the number of nodes that power up, and the duration of each call is also exponentially distributed.

Routing Protocols Implementation - By avoiding the need to perform simulation at the packet level, routing implementation is simplified. When a new route request arrives or a route is broken due to mobile hosts' migration, the source node will broadcast a route query message, and all nodes that may receive and forward it will consume the same amount of energy. Five route selection schemes are implemented in our simulator. The first one uses a minimum hop metric, the second selects a route according to the stability of the route [10], the third uses a minimum battery cost metric, the fourth uses a min-max battery cost metric, and the last one uses the conditional max-min battery capacity scheme. 


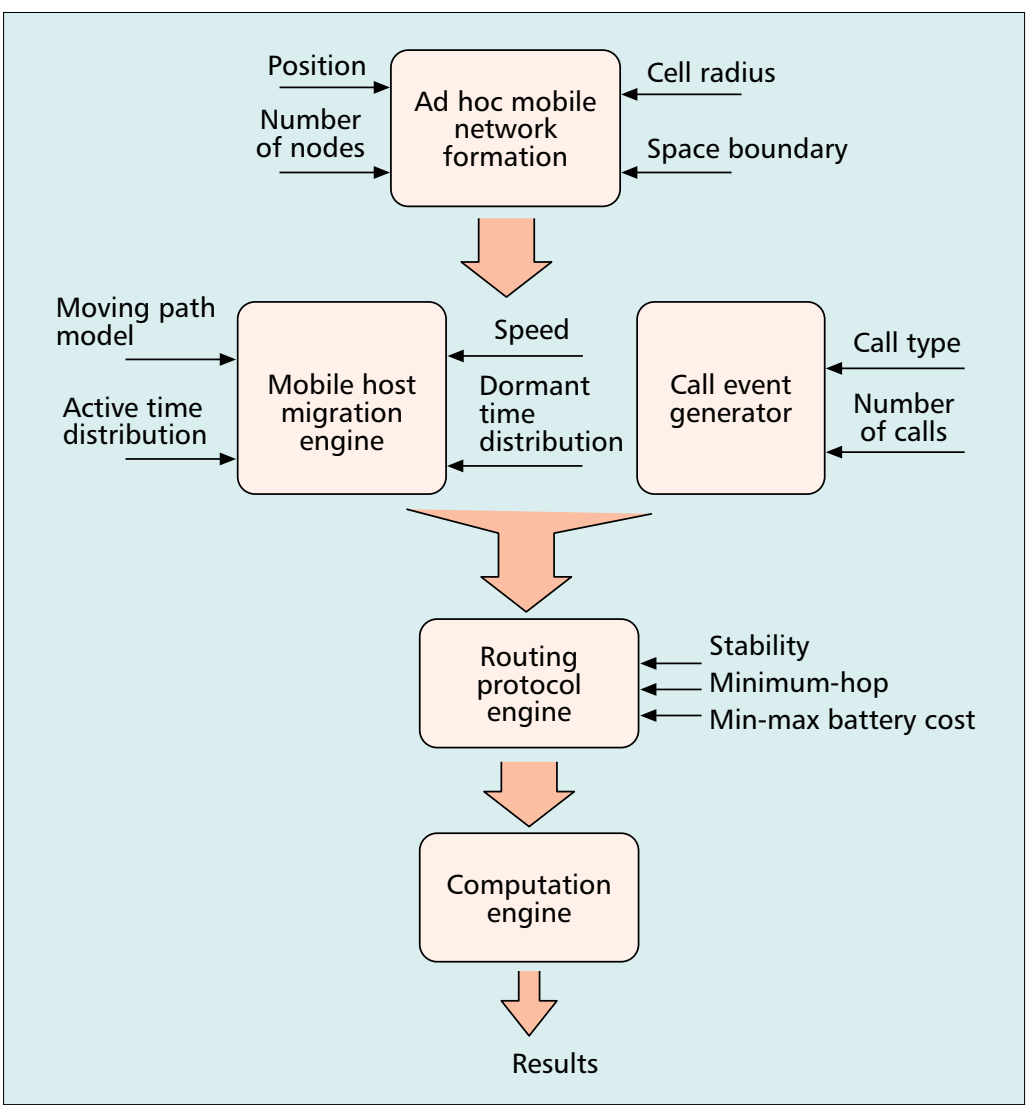

Figure 2. An ad hoc mobile network simulation model.

Power Consumption Computation - At the end of each simulation time slot, energy consumed by each node during this period is computed. We classify power consumption of a mobile host into two categories:

- Communication-related

- Non-communication-related

Power drained from the former is proportional to the amount of traffic transmitted or relayed by the node. We assume that the power consumption rate for the latter is fixed. In reality, this may fluctuate from time to time. This demands further investigation.

According to different types of networks for different applications, the ratio of the power used for these two parts can be quite different. This ratio is defined by

$$
\sigma=\frac{\text { Power }_{C R}}{\text { Power }_{N C R}},
$$

where the numerator refers to the maximum power for the communication-related part and the denominator refers to the non-communication-related part.

To evaluate the impact of different route selection schemes for the lifetime of a mobile host in different networks, three special cases are considered in our simulations:

- The power consumption rate for communication-related applications is much larger than non-communication-related.

- Both parts are on the same order.

- The power consumption rate for non-communication-related part is much larger.

All nodes are assumed to have the same amount of battery capacity at the beginning of simulation. The simulation stops when only two nodes are alive because at this instance, source and destination nodes are at most one hop away.

\section{Simulation RESULTS}

In our simulations, five different route selection schemes are considered:

- Minimum total transmission power

- Minimum battery cost

- Min-max battery cost

- Conditional max-min battery capacity

- Association stability

We assume the transmission power of each node is the same and fixed. Therefore, the minimum total transmission power scheme is the same as

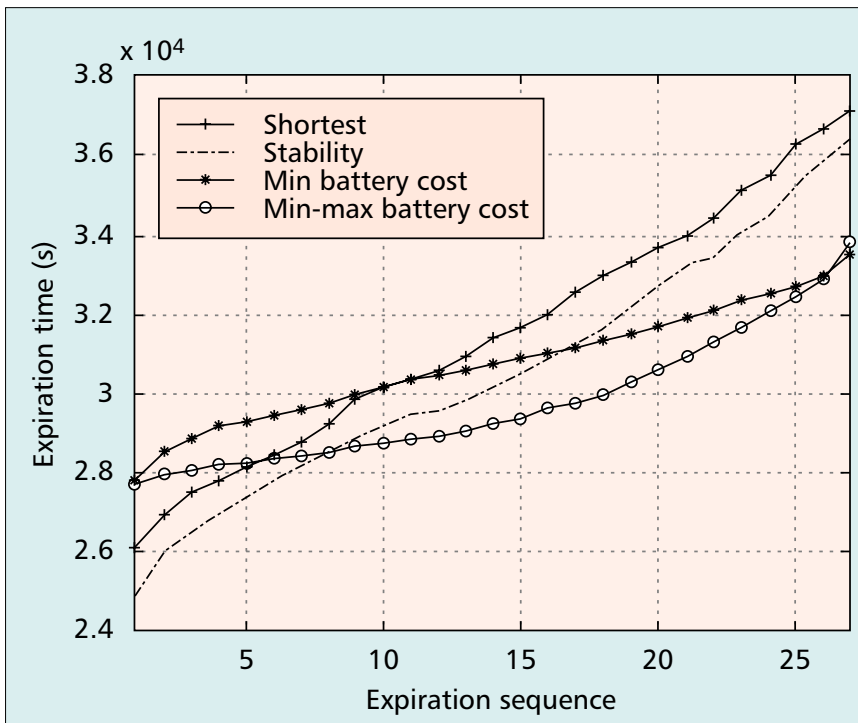

(a)

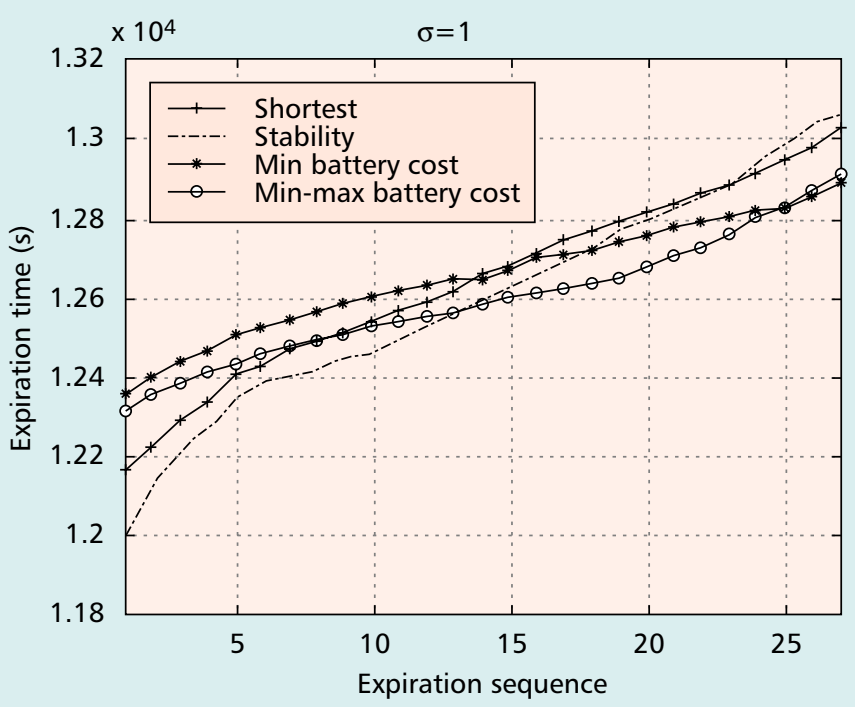

(b)

Figure 3. a) Expiration time vs. expiration sequence when $\sigma=10 ; b)$ expiration time vs. expiration sequence when $\sigma=1$. 
the standard shortest path algorithm. The battery cost function is defined by the following equation [8], whose value is inversely proportional to the remaining battery capacity:

$$
f_{i}\left(c_{i}^{t}\right)=\frac{1}{c_{i}^{t}} .
$$

Since a host can forward packets only when its battery capacity is above zero, the value of the cost function will always be finite.

The route stability scheme selects routes that are long-lived. ${ }^{4}$ We record the expiration time of each node (i.e., the time when a node exhausts its battery capacity), and also note the node expiration sequence - which is sorted according to expiration time. The first node in this sequence has the shortest expiration time and the last node the longest. Simulation for each method is repeated 10 times, and the average value is recorded.

In Fig. 3a four route selection schemes are compared when $\sigma=10$ :

- Shortest path (SP)

- Minimum battery cost (MBC)

- Min-max battery cost (MMBC)

- Route stability (RS)

The time of the first node exhausting its battery is much earlier than that of the last node for SP and RS schemes. Since these metrics do not take the battery capacity of each node into consideration, some nodes will tend to have higher relaying load, which results in a widely differing power consumption profile for each node.

Consider the network shown in Fig. 4. Node 6 will be selected as the relaying node for routes $0-3,1-4$, and $2-5$ if the SP routing scheme is applied. As a result, node 6 will have a high power consumption rate and hence a short lifetime. A similar situation happens to the stable node in the route stability metric.

However, it should be noted that by applying the SP scheme, most nodes have a longer expiration time. This is because it will find shorter routes than the others. For a specified amount of traffic, since each communication goes through less hops, the average relaying load for each node is reduced and the lifetime prolonged. In our simulations, the average route length ${ }^{5}$ for $\mathrm{SP}, \mathrm{RS}, \mathrm{MBC}$, and MMBC is $3.03,3.58,3.24$, and 3.48 hops, respectively. The MBC scheme finds shorter routes than the MMBC scheme. Therefore, all nodes have longer lifetimes. Nonetheless, because of the problem illustrated in Fig. 1, the time to the first node failure is almost the same in both cases.

As the value of $\sigma$ decreases (meaning power used by communication-related applications is reduced), the impact of this part on the lifetime of a mobile host is less significant. Hence, results among these metrics do not differ as much as those in the first case, as shown in Fig. 3b when

${ }^{4}$ This is different from the ABR routing protocol [9],

which also takes relaying load into consideration.

${ }^{5}$ The average route length is a function of the network size and connectivity, and we measure this value only when all nodes are "alive."

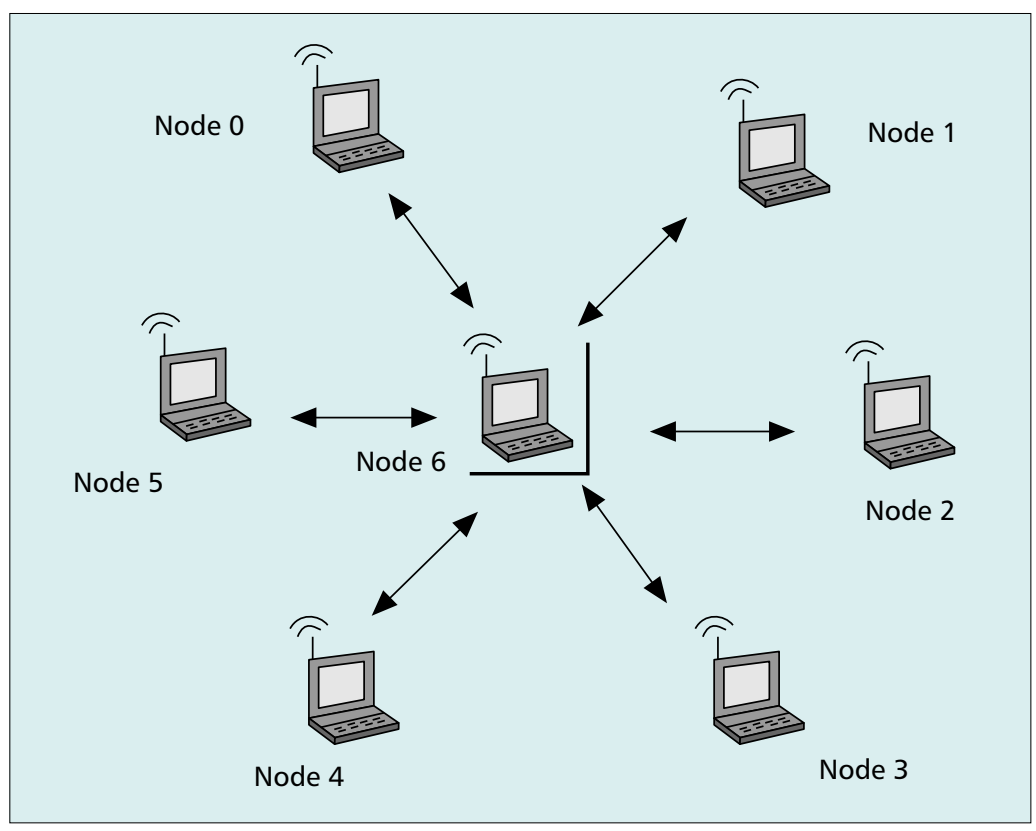

Figure 4. An illustration of the shortcoming in minimum-hop routing.

$\sigma=1$. The maximum difference among the curve of the RS scheme and those of battery cost schemes is only 2.4 percent. For the SP scheme, it is only 1.3 percent. We observe that if $\sigma=0.1$, all nodes "die" at almost the same time for all route selection schemes because in that case, the lifetime of a node is dominated by the power consumption rate of non-communication-related applications, which is the same for all nodes.

Since the performance of the CMMBCR scheme is a function of $\gamma$, different values of $\gamma$ (between 0 and 100) are examined. The mean and standard deviation of the lifetime of all nodes are used to evaluate the influence of $\gamma$. The expiration time vs. expiration sequence for different values of $\gamma$ is illustrated in Fig. 5. When $\gamma=0$, there is no protection margin, and this metric is identical to the SP scheme.

As the value of $\gamma$ increases, nodes will be protected at the early stage before they exhaust their battery capacity (i.e., their batteries will be more fairly used), and therefore, the standard deviation of nodes' lifetime decreases (Fig. 6b). Since the probability that longer paths will be selected increases (which implies the relaying load for each node increases), the average lifetime of all nodes decreases as $\gamma$ increases, as shown in Fig. $6 \mathrm{a}$. The average route length occurring over different values of $\gamma$ is shown in Fig. 7 .

Figures $6 \mathrm{a}$ and $6 \mathrm{~b}$ also reveal that it is not feasible to use each node's battery capacity fairly while trying to maximize the lifetime of most nodes in the network. One must do a trade-off between these two goals. If all nodes in the network are equally important and no node must be overused more than the others, a higher value of $\gamma$ is preferred. On the contrary, if the loss of a few nodes is acceptable, a lower value of $\gamma$ should be used to extend the lifetime of nodes in the network. The actual value of $\gamma$ will therefore depend on network size and the mobility profile of each node. These considerations can be used as part of system and protocol design. 


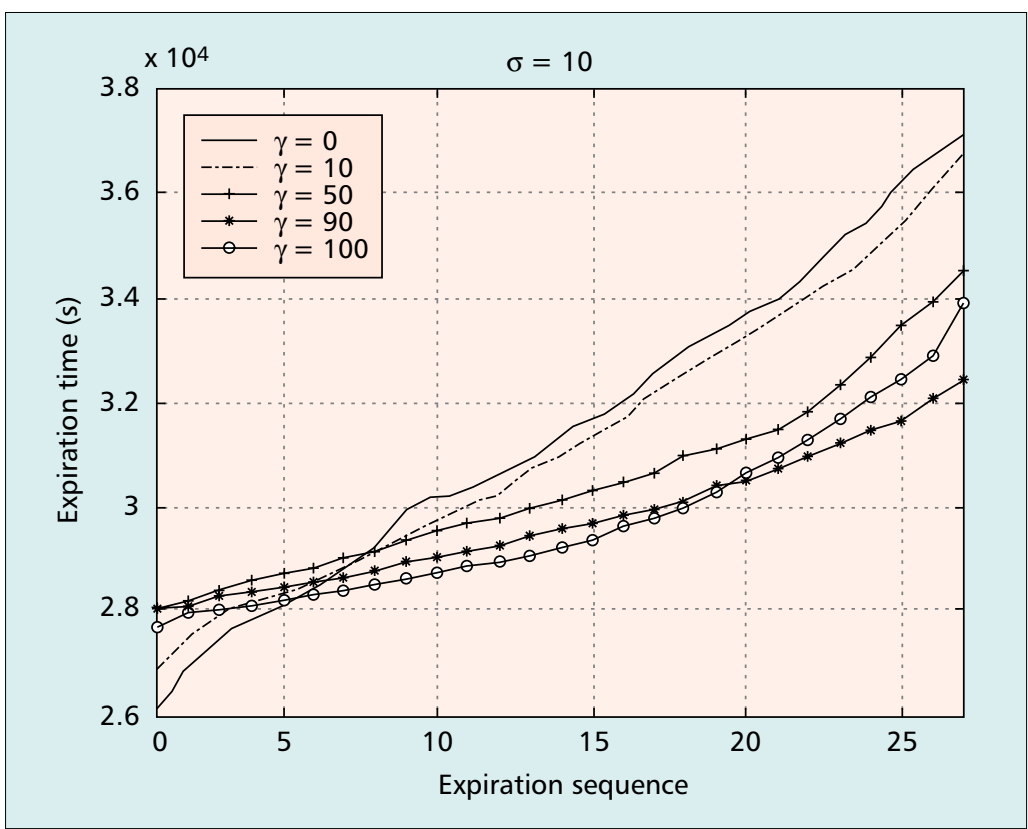

Figure 5. Expiration time vs. expiration sequence for different values of $\gamma$ when $\sigma=10$. consumption, stability of routes, and so on should be considered as well. From our simulation results, we discover that if nodes in an ad hoc wireless network expend most of their power on communication-related applications, poweraware routing protocols, like minimum battery cost and min-max battery cost schemes, can prevent nodes from being unwisely overused. This extends the time until the first node powers down and increases the operation time before the network is partitioned. However, these power-aware routing protocols tend to select longer paths, which increases the average relaying load for each node and therefore reduces the lifetime of most nodes.

Our investigations reveal that these two goals (to use each node fairly and extend their lifetimes) are not compatible. A trade-off between them is needed. Our proposed conditional maxmin battery capacity routing (CMMBCR) scheme chooses a shortest path if all nodes in all possible routes have sufficient battery capacity. When the battery capacity for some nodes goes below a predefined threshold $(\gamma)$, routes going through these nodes will be avoided, and therefore the time until the first node power-down is extended.

By adjusting the value of $\gamma$, we can maximize either the time when the first node powers down or the lifetime of most nodes in the network. It should be noted that if the power used for a communication subsystem only takes a small portion of the overall host power consumption, the difference of performance in power efficiency is negligible for all routing protocols, regardless of the types of routing metrics considered.

\section{REFERENCES}

[1] E. Royer and C.-K. Toh, "A Review of Current Routing Protocols for Ad Hoc Mobile Wireless Networks," IEEE Pers. Commun., vol. 6, no. 2, Apr. 1999, pp. 46-55.

[2] S.-J. Lee, M. Gerla, and C.-K. Toh, "A Simulation Study of Table-Driven and On-Demand Routing Protocols for Mobile Ad Hoc Networks," IEEE Network, July 1999.

[3] C.-K. Toh, "Associativity Based Routing for Ad Hoc Mobile Networks," Wireless Pers. Commun. J., Special Issue on Mobile Networking and Computing Systems, vol. 4, no. 2, Mar. 1997.

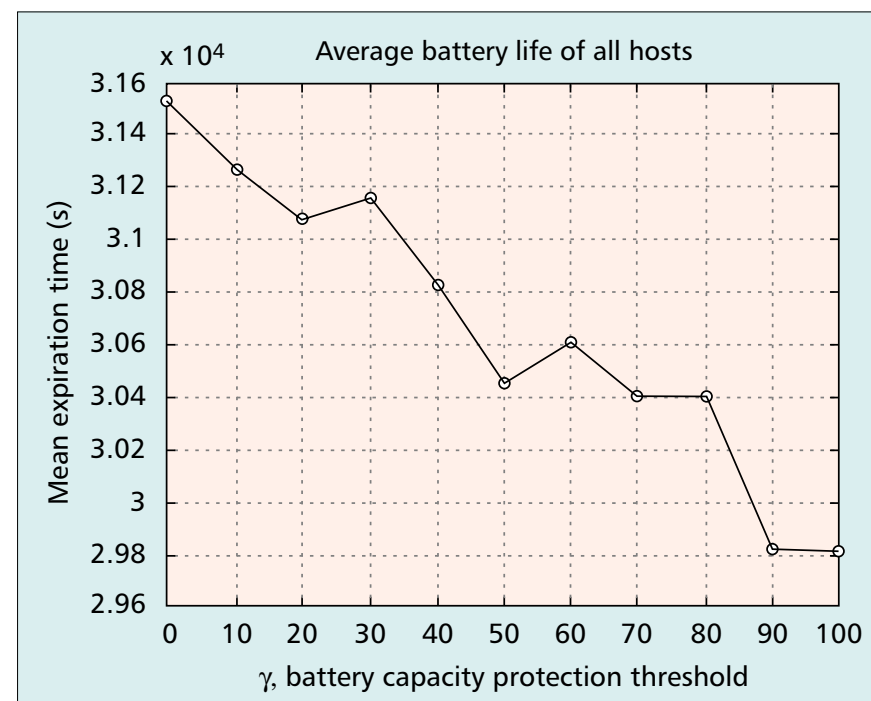

(a)

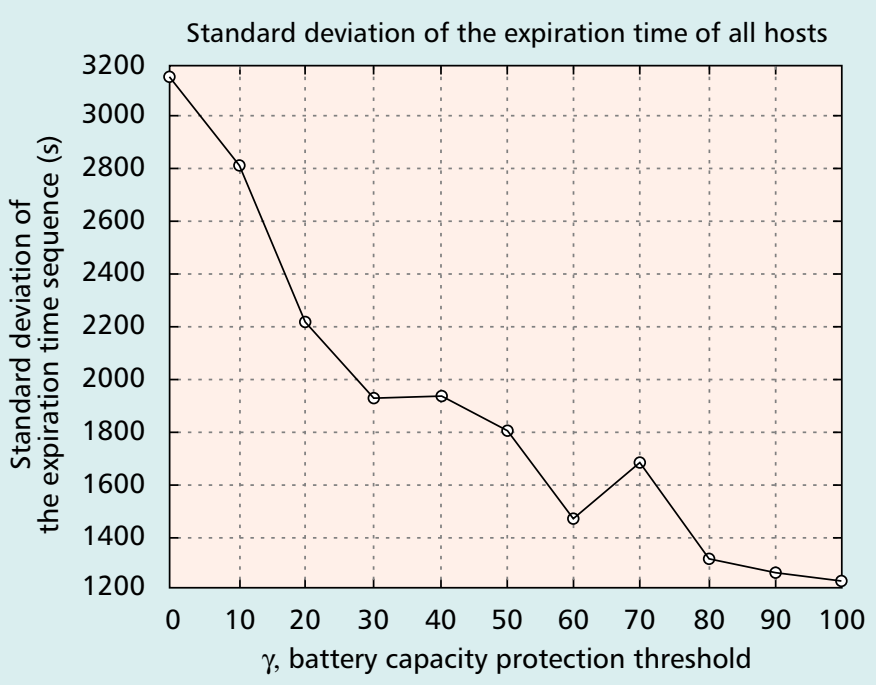

(b)

Figure 6. a) Average host lifetime vs. increasing $\gamma$; ) standard deviation of lifetime of all nodes vs. $\gamma$. 
[4] P. Latteieri, C. Schurgers, and M. B. Srivastava, "Adaptive Link Layer Strategies for Energy Efficient Wireless Networking," ACM WINET.

[5] A. Alwan et al., "Adaptive Mobile Multimedia Networks," IEEE Pers. Commun., Apr. 1996.

[6] S. Singh and C. S. Raghavendra, "PAMAS-Power Aware Multi-Access protocol with Signaling for Ad Hoc Networks," ACM Commun. Rev., July 1998.

[7] K. Scott and N. Bambos, "Routing and Channel Assignment for Low Power Transmission in PCS," Proc. ICUPC '96, vol. 2, pp. 498-502, Oct. 1996.

[8] S. Singh, M. Woo, and C. S. Raghavendra, "PowerAware Routing in Mobile Ad Hoc Networks," Proc. MobiCom '98, Dallas, TX, Oct. 1998.

[9] Z. J. Haas, "A New Routing Protocol for the Reconfigurable Wireless Networks," Proc. ICUPC '97, San Diego, CA, Oct. 1997

\section{AdDITIONAL READING}

[1] V. D. Park and M. S. Corson, "A Highly Adaptive Distributed Routing Algorithm for Mobile Wireless Networks," IEEE INFOCOM '97, Kobe, Japan, 1997.

[2] C. E. Perkins and E. M. Royer, "Ad-Hoc On-Demand Distance Vector Routing," Proc. 2nd IEEE Wksp. Mobile Comp. Sys. and Apps., Feb. 1999.

[3] D. Dube et al.," Signal Stability-Based Adaptive Routing (SSA) for Ad Hoc Mobile Networks," IEEE Pers. Commun., Feb. 1997, pp. 36-45.

[4] V. Rodoplu and T. H.-Y. Meng, "Minimum Energy Mobile Wireless Networks," Proc. Int'l Conf. Commun. June 1998.

\section{BIOGRAPHY}

C.-K. TOH [SM] (cktoh@ece.gatech.edu) received his B.Eng. and Ph.D. degrees in electrical engineering and computer science from Manchester and Cambridge Universities, respectively. He authored the book Wireless ATM and Ad Hoc Networks (Kluwer Academic, 1996). He is an editor for IEEE JSAC, IEEE Network, Journal on Communications Networks, and Journal on Wireless Information Networks. He received the KICS Appreciation Award and ACM Recognition of Service Award in 2000. He is listed in MARQUIS Who's Who in the World of Science and Engineering. Currently, he is a faculty member at Georgia Tech and serves as Chair of IEEE TCPC Subcommittee on Ad Hoc Mobile Wireless Networks. He is Technical Chair for IEEE Symposium on Ad Hoc Mobile Networks. and Technical Vice Chair for IEEE Wireless Communications and Networking Conference (WCNC 2002). He is a life fellow of the Cambridge Philosophical Society.

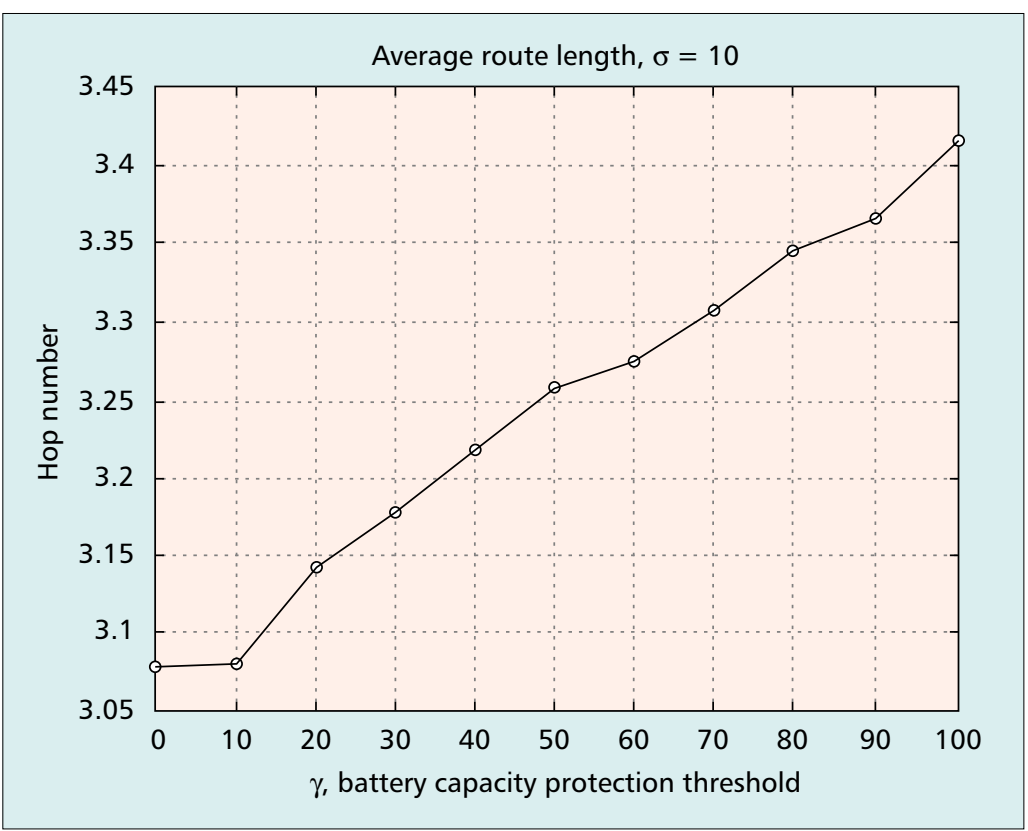

Figure 7. Average route length $v$ s. different $\gamma$. 